Case Report

\title{
Delayed Small Bowel Ischemia following Minor Mesenteric Injury
}

\author{
Gil Hwan Kim a,b, Jae Hun Kim a,b,*, Sang Bong Lee a,b \\ a Department of Trauma and Surgical Critical Care, Pusan National University Hospital, Busan, Korea \\ ${ }^{b}$ Biomedical Research Institute, Pusan National University Hospital, Busan, Korea
}

\section{Article history:}

Received: August 11, 2017

Revised: September 18, 2017

Accepted: September 18, 2019

\author{
${ }^{*}$ Corresponding Author: \\ Jae Hun Kim \\ Department of Trauma and Surgical \\ Critical Care, Pusan National \\ University Hospital, Busan, Korea \\ E-mail: jjangmdkmdk@hanmail.net \\ ORCID \\ https://orcid.org/0000-0003-4504-9898
}

\section{ABSTRACT}

We report a case of delayed presentation of small bowel ischemia following minor mesenteric injury after blunt abdominal trauma. Traumatic small bowel and/or mesenteric injury is rare, and minor mesenteric injury is usually managed conservatively. However, mesenteric injury may cause potentially fatal conditions such as hemorrhages or peritonitis in extremely rare cases and require laparotomy. We present a case of small bowel ischemia that occurred 3 days after minor mesenteric injury from blunt abdominal trauma.

Keywords: traffic accidents, abdominal injuries, laparotomy

\section{Introduction}

After the liver and spleen, the small bowel and/or its mesentery is the third most commonly injured organs from blunt abdominal trauma. However, the incidence of these injuries is rare and timely diagnosis is challenging [1]. Seatbelt injury from motor vehicle accidents is the most common and well-described cause of small bowel and mesenteric injury [2]. Minor mesenteric injury is usually self-limited and conservatively managed. However, mesenteric injury can lead to fatal hemorrhage or peritonitis in extremely rare cases and may require a laparotomy. We report a rare case of delayed small bowel ischemia without perforation following minor mesenteric injury after blunt abdominal trauma.

\section{Case Report}

A 56-year-old man presented to our trauma center after a traffic accident. He was a driver and had fastened a seatbelt. He was alert, vital signs were stable, and there was no seatbelt mark sign on his abdomen. His abdomen was soft and not distended, but there was localized right upper quadrant tenderness. His only complaint was pain in the left hip. A left acetabular fracture with hip dislocation was found in plain X-ray and computed tomography (CT) of the hip. CT of the abdomen with contrast showed minimal mesenteric fat infiltration without free peritoneal fluid (Figure 1). After closed hip reduction in the emergency room, elective surgery was planned for hip dislocation and left acetabular fracture. He was admitted to the intensive care unit for close observation 


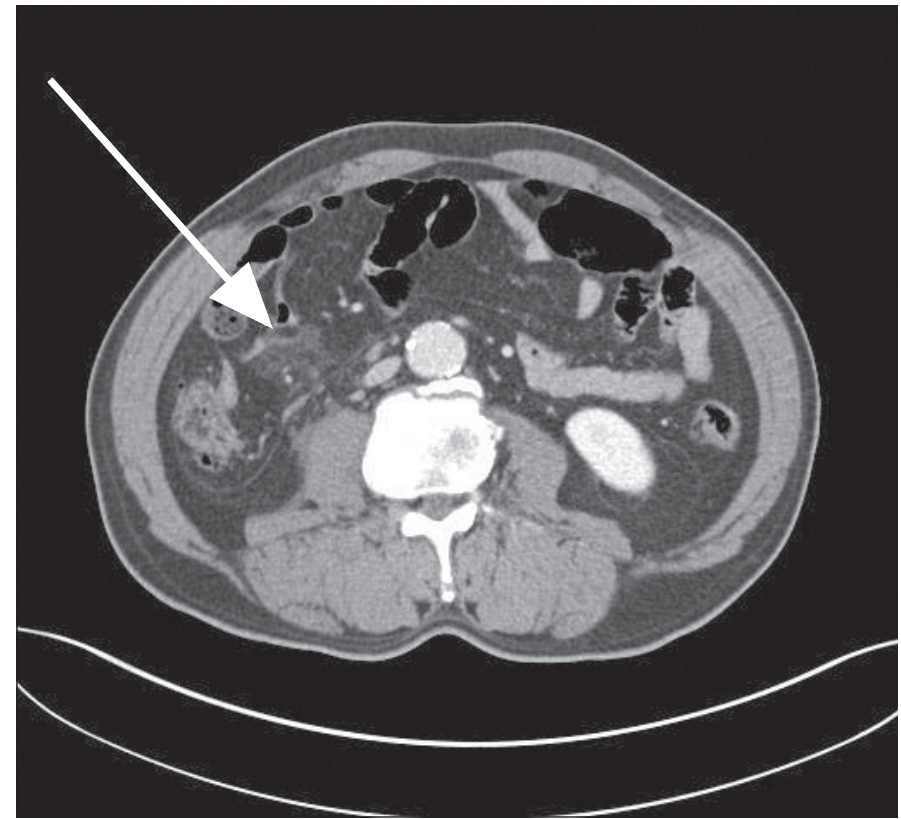

Figure 1. Minimal mesenteric fat infiltration without intraperitoneal free air or fluid (arrow).

of the mesenteric contusion, which can develop to a delayed bowel injury. Repeated abdominal CT was performed on the first day empirically, because the presence of delayed intestinal injury can be suspected in terms of mesenteric contusion with localized tenderness. There were no findings which indicating bowel injury, such as intra-abdominal free air.

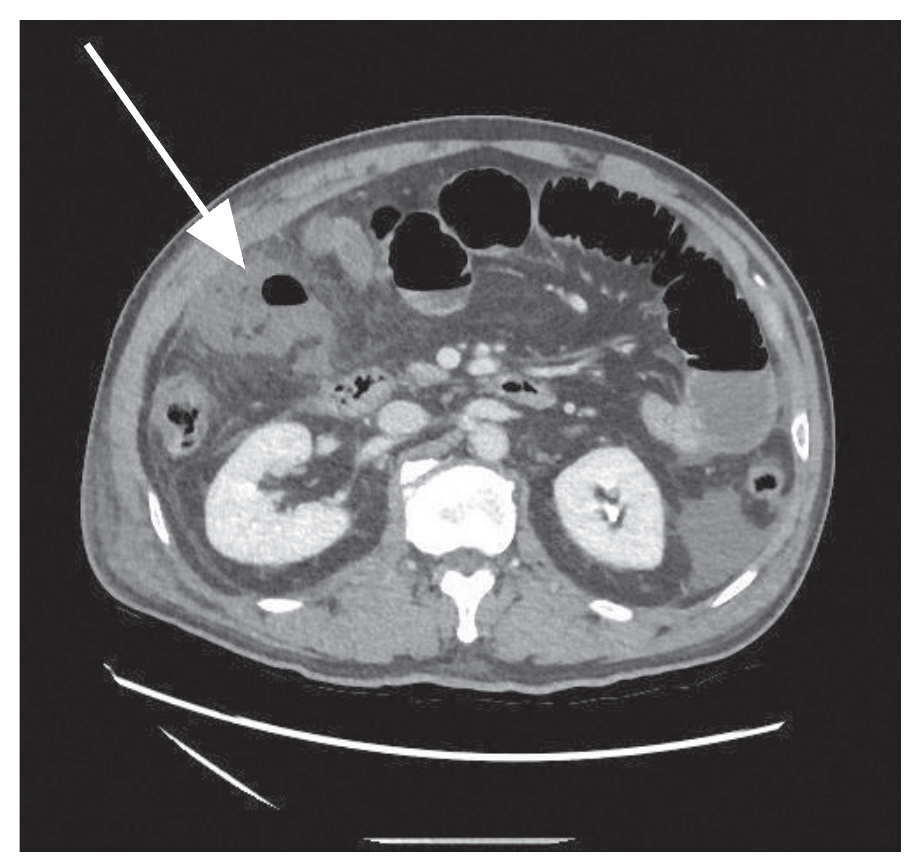

Figure 2. Repeated computed tomography scan after 3-day posttrauma, showing decreased small bowel mucosal enhancement with intraperitoneal free fluid (arrow).

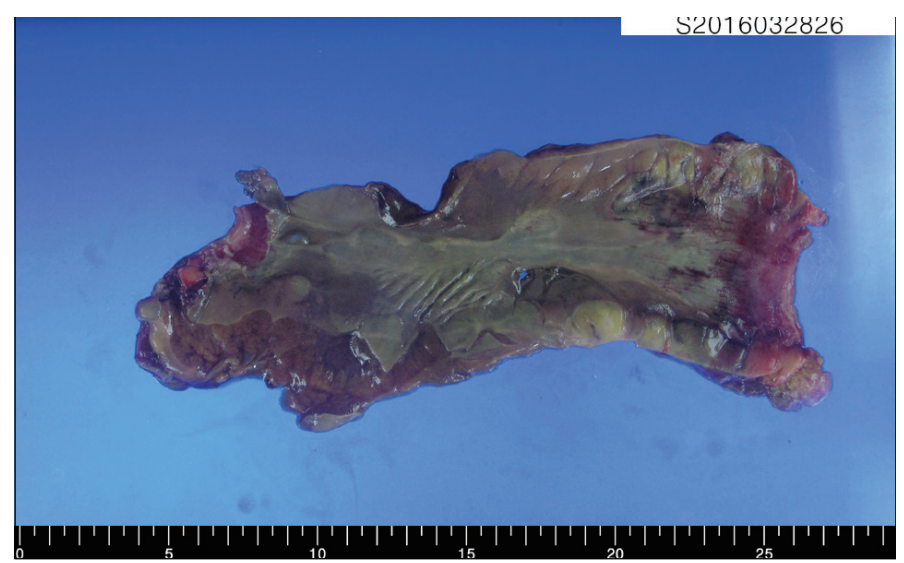

Figure 3. Postoperative specimen of the resected distal ileum showing transmural infarction.

Three days after the trauma, the patient underwent hip surgery. Postoperatively, he complained of abdominal pain with abdominal distension. The localized right upper quadrant tenderness became worse, but there was no generalized tenderness observed. He also developed new-onset fever. Although laboratory findings including white blood cell count were in normal ranges, repeated abdominal CT was performed because of the changing abdominal physical examinations. Abdominal CT demonstrated small bowel ileus, increased intraperitoneal free fluid without solid organ injury and decreased enhancement of the small bowel mucosa (Figure 2). Under suspicion of bowel injury, exploratory laparotomy was performed. Intraoperative findings included an ileocolic mesenteric tear and ischemic change of the distal ileum (Figure 3). The patient underwent segmental resection and anastomosis of the small bowel.

\section{Discussion}

Small bowel and/or mesenteric injury after blunt abdominal trauma is rare, occurring in $<1 \%$ of all injuries, despite being the third most common injury among the abdominal organs. Because it is associated with high mortality and morbidity, timely diagnosis and surgical intervention should not be delayed. Delayed diagnosis may lead to serious hemorrhage or peritonitis even from minor injuries [3].

The most common cause of small bowel and mesenteric injury is motor vehicle accident, especially with a fastened seatbelt. The presence of a seatbelt mark sign is a reliable predictor of bowel injury. The mechanism involves crushing the gastrointestinal tract between the vertebra and anterior abdominal wall by direct force, shearing force between fixed and mobile portions of the small bowel by deceleration and 
increasing intraluminal pressure of the bowel [2].

Mesenteric injury can lead to severe complications, such as life-threatening hemorrhage from disruption of mesenteric vessels and peritonitis from intestinal ischemic necrosis [4]. Direct injury mechanisms alone are insufficient to explain delayed small bowel injury that develops a few days or weeks after the trauma. In our case, the direct force itself was not expected to be large, considering that there was no seatbelt mark sign. Intestinal ischemia seems to be a secondary injury resulting from mesenteric blunt trauma rather than direct ischemia of the bowel wall. Although the minor mesenteric injury may show a normal clinical presentation initially, it can affect blood supply to the small bowel and cause ischemia, mucosal ulceration, and submucosal inflammation of adjacent bowel segments [5,6].

CT has a major role in the diagnosis of small bowel injury in stable patients with blunt trauma. Some findings indicate mesenteric injury, such as mesenteric hematoma, infiltration, free abdominal fluid, and active blood extravasation [7]. However, the false-negative rate in CT diagnosis of small bowel injury is approximately $13 \%$ [1]. In particular, delayed small bowel perforation or ischemia is more difficult to diagnose at the appropriate time.

We suggest that when conservatively treating blunt abdominal trauma patients, close observation is necessary with repeated physical examination and timely diagnosis using abdominal CT. To do so, physicians should always be suspicious of delayed small bowel injury, including ischemia or perforation, even in the case of minor injury.

\section{Conflicts of Interest}

No potential conflict of interest relevant to this article was reported.

\section{Acknowledgments}

This work was supported by clinical research grant from Pusan National University Hospital in 2018.

\section{References}

[1] Fakhry SM, Watts DD, Luchette FA; EAST Multi-Institutional Hollow Viscus Injury Research Group. Current diagnostic approaches lack sensitivity in the diagnosis of perforated blunt small bowel injury: analysis from 275,557 trauma admissions from the EAST multi-institutional HVI trial. J Trauma 2003;54(2):295-306.

[2] Iaselli F, Mazzei MA, Firetto C, D'Elia D, Squitieri NC, Biondetti PR, et al. Bowel and mesenteric injuries from blunt abdominal trauma: a review. Radiol Med 2015;120(1):21-32.

[3] Xeropotamos NS, Nousias VE, Ioannou HV, Kappas AM. Mesenteric injury after blunt abdominal trauma. Eur J Surg 2001;167(2):106-9.

[4] Luo T, Lu Z, Zhi K, Zhang H, Xue X, Pang T, et al. Surgical experience of delayed intestinal necrosis following blunt abdominal trauma: 19 consecutive cases in 10 years. Int J Clin Exp Med 2016;9(2):4083-8.

[5] Hamidian Jahromi A, Johnson L, Youssef AM. Delayed small bowel perforation following blunt abdominal trauma: a case report and review of the literature. Asian J Surg 2016;39(2):109-12.

[6] Kang GH, Jeon TJ, Seo DD, Oh TH, Kim SH, Cho HS, et al. Ileal stenosis occurred 3 months after blunt abdominal trauma. Korean J Gastroenterol 2011;57(6):370-3.

[7] Murakami R, Tajima H, Kumazaki T, Kobayashi Y. CT findings of mesenteric injury after blunt trauma. CMIG Extra Cases 2004;28(2):11-4. 